\title{
ENGINEERING OF THE AGS SNAKE COIL ASSEMBLY*
}

\author{
M. Anerella $a^{\#}$ R. Gupta, P. Kovach, A. Marone, S. Plate, K. Power, J. Schmalzle, E. Willen, \\ Brookhaven National Laboratory, Upton, NY 11973 USA
}

\begin{abstract}
A $30 \%$ Snake superconducting magnet is proposed to maintain polarization in the AGS proton beam, the magnetic design of which is described elsewhere [1]. The required helical coils for this magnet push the limits of the technology developed for the RHIC Snake coils. First, fields must be provided with differing pitch along the length of the magnet. To accomplish this, a new 3-D CAD system ("Pro/Engineer" from PTC), which uses parametric techniques to enable fast iterations, has been employed. Revised magnetic field calculations are then based on the output of the mechanical model. Changes are made in turn to the model on the basis of those field calculations. To ensure that accuracy is maintained, the final solid model is imported directly into the CNC machine programming software, rather than by the use of graphics translating software. Next, due to the large coil size and magnetic field, there was concern whether the structure could contain the coil forces. A finite element analysis was performed, using the 3-D model, to ensure that the stresses and deflections were acceptable. Finally, a method was developed using ultrasonic energy to improve conductor placement during coil winding, in an effort to minimize electrical shorts due to conductor misplacement, a problem that occurred in the RHIC helical coil program. Each of these activities represents a significant improvement in technology over that which was used previously for the RHIC snake coils.
\end{abstract}

\section{A PROCESS FOR MODELING AGS SNAKE HELICAL COILS}

Previously, helical coil grooves or blocks were created through a time-consuming process using CAD software. After defining the helical path of each groove, numerous cross-sections of the groove were constructed at intervals along the paths. All cross-sections were manually oriented normal to their helical path during construction. Surfaces were then lofted through these cross-sections and used to perform the cuts necessary to produce the grooves in the basic cylindrical part. This process made changes quite difficult. Where variable pitch helixes were required, separate helixes were constructed and joined.

With the use of Pro/Engineer, the process of creating variable pitch helical grooves changed dramatically. Using PTC's Advanced Surfacing module with the Pro/E
Foundation module, the complex helical cuts are produced in a more efficient manner. Also, since Pro/E can easily construct variable pitch helixes (see Figure 1) the process of creating the helical paths was greatly simplified. The paths produced in this manner were parametric, meaning they could easily be changed without the need to reconstruct them as the design evolved.

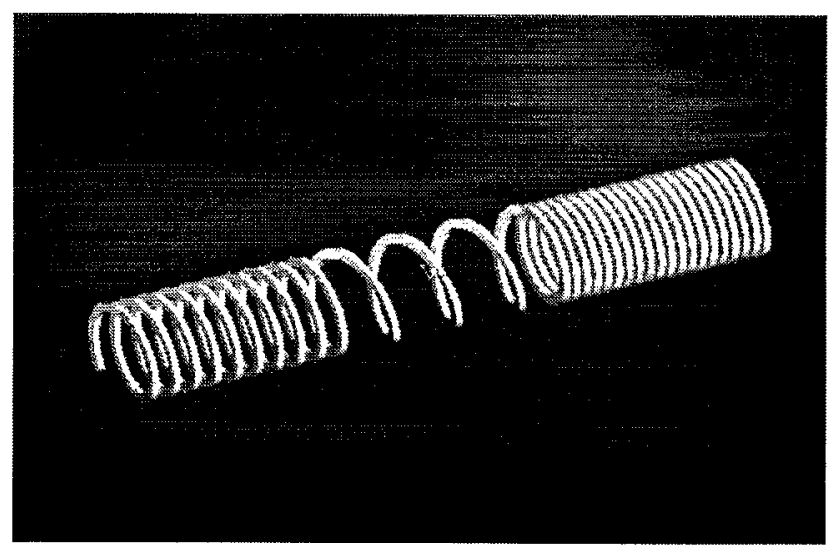

Figure 1.

A technique was developed to construct the variable pitch cuts in the tube. Helical surfaces were constructed normal to a cylindrical surface and then intersected with that surface to produce the curves that define the helical portions of the grooves' paths. Various techniques were used to develop the paths of the coil ends. The end curves along with the helical curves define the basic paths of the grooves (see Figure 2). These basic curves were then projected onto a larger cylindrical surface to produce the remaining curves required to define the variable crosssection sweeps. The completed cuts were then produced using the Pro/Engineer variable section sweep cut functionality (see figure 3 ).

In the coil models all grooves were defined and created in this manner. A typical, completed coil tube produced using this process is shown in Figure 4. Since most of the geometry created using this process is parametric, design changes that would normally require extensive geometry modifications could frequently be accomplished by changing a single parameter, resulting in all subsequent geometry being automatically updated. In an evolving, iterative design such as this, considerable time has been saved as a result of these capabilities.

\footnotetext{
*Work supported by the U.S. Department of Energy under Contract No. DE-AC02-98CH10886.

\#mda@brbl.gov
} 


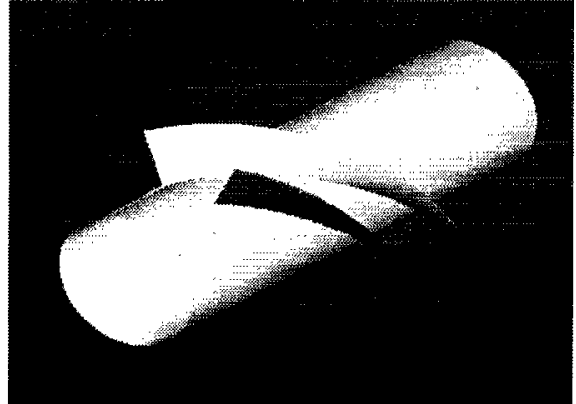

Figure 2.

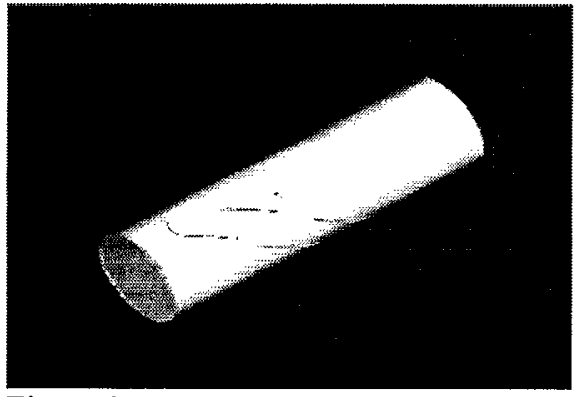

Figure 3.

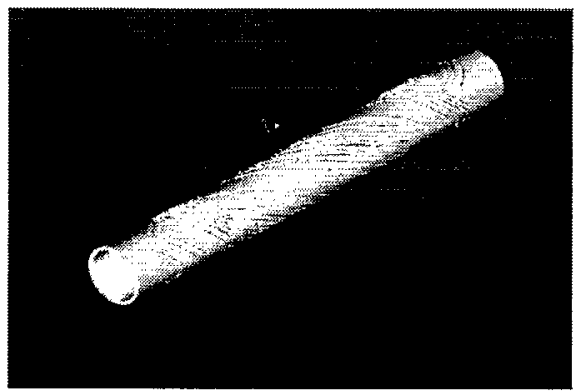

Figure 4.

Once the final magnetic and mechanical designs had been established and verified, the models of the two coil support tubes were sent electronically to the machine tool, using NC programming software and post processors developed by both PTC and MasterCam. In this way, tool paths were developed directly from the engineering surfaces, and not as interpreted by IGES or other graphics translation software. Parts were subsequently machined using a 4-axis CNC machine tool and inspected to be within $0.25 \mathrm{~mm}$ of desired surface position along all locations of the helices. The inner coil support tube in the machine tool is shown below in figure 5 .

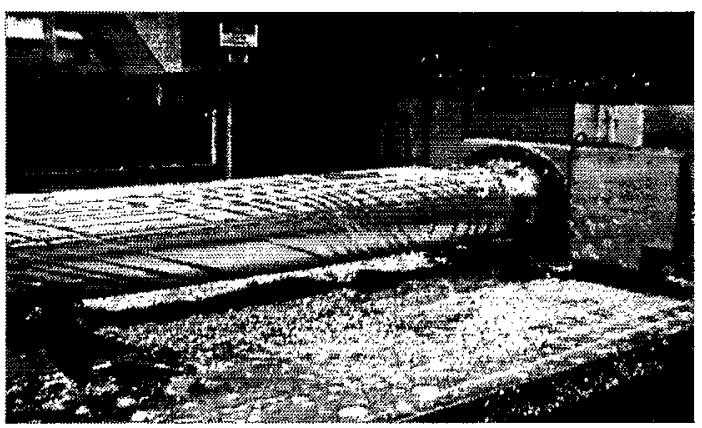

Figure 5.

\section{AGS SNAKE COIL TUBE ANALYSIS}

A three-dimensional finite element structural analysis was done on the inner and outer coil support tubes for the AGS snake magnet to determine the stress and deflections in each tube under the Lorentz forces. A 30 inch long section from the center of each tube was used for the analysis. The calculated azimuthal pressures exerted by each coil block were scaled based on the percentage of the groove depth occupied by the coil windings. The resulting pressure for each block as indicated in Table 2 was applied the full sidewall of the groove. Both ends of the tube were completely constrained and the outside diameter of the tube was constrained in the radial direction. Material was assumed to be aluminum with a Young's modulus of $10 \mathrm{e} 6 \mathrm{psi}$. Results shown in figures 6 through 9 indicate a peak stress of 34000 psi for the inner tube and 13000 psi for the outer tube with maximum deflections of .0020 inches and .0016 inches respectively.

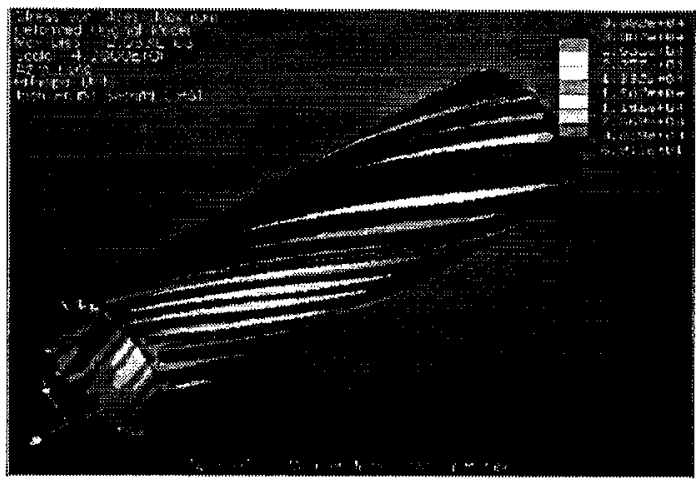

Figure 6. Inner Tube Stress

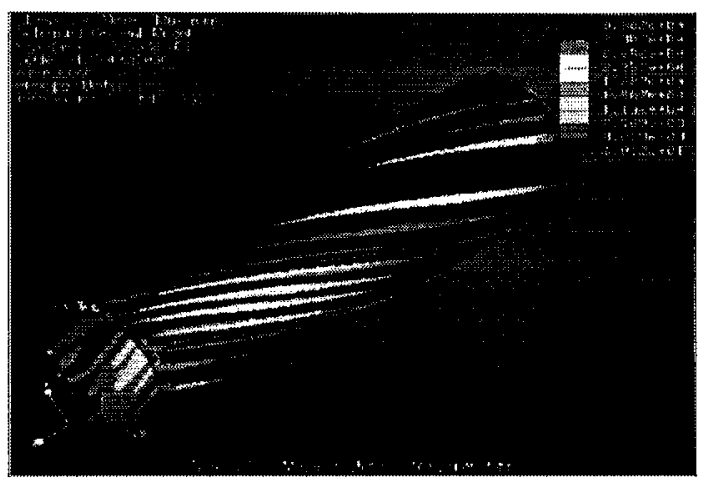

Figure 7. Inner Tube Deflection

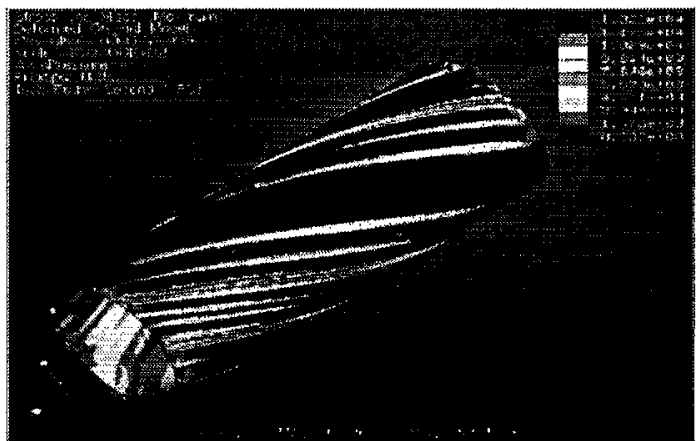

Figure 8. Outer Tube Stress 


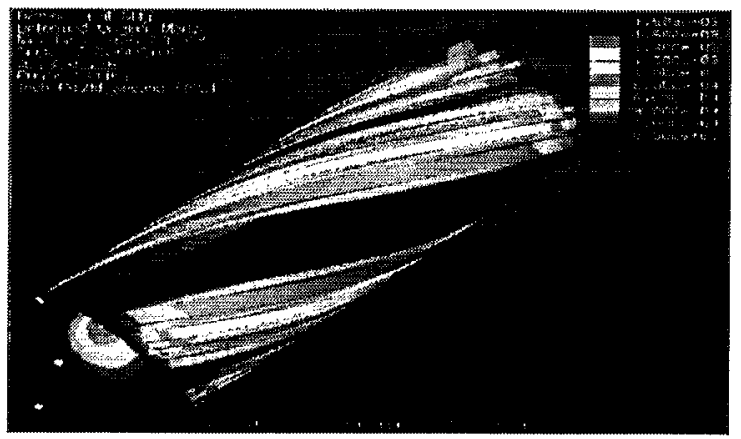

Figure 9. Outer Tube Deflection

Table 1. Tube Dimensions (in.)

\begin{tabular}{|lllll|}
\hline Tube & ID & OD & $\mathrm{t}$ & slot depth \\
\hline Inner & 7.70 & 9.04 & .67 & .512 \\
Outer & 9.76 & 11.10 & .67 & .512 \\
\hline
\end{tabular}

Table 2. Lorentz Force Loads

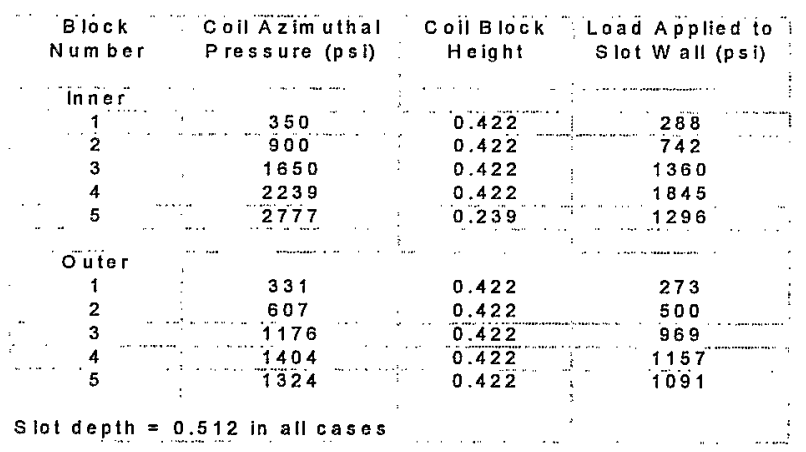

\section{A METHOD TO USE ULTRASONIC ENERGY TO IMPROVE CONDUCTOR PLACEMENT DURING COIL WINDING}

AGS Snake coils are arranged in blocks of windings within the machined tube grooves. These winding blocks are arrays of round cable, twelve rows wide and nine layers high as shown in Figure 10. Between each vertical layer is a b-stage epoxy impregnated fiberglass substrate. After winding, the cables are compressed into the substrate during an elevated temperature and pressure cure cycle. The cure cycle seats the conductors into the substrate while the epoxy flows to eliminate voids, thereby providing suitable support for the conductors.

Inherent in the helical winding pattern and the inward shift in radial position described above is a surplus of wire placed into the grooves during winding. It was found during production of the RHIC helical coils that this excess conductor led to electrical shorts in several instances due to the resulting incorrect positioning of conductor, as is seen in Figure 11. In the AGS Snake application this problem would become more severe. Therefore, a method was developed using a 500-watt hand-held ultrasonic welder to seat the conductors into the substrate below during the winding process. This process was used after each of the nine layers of conductors was placed into the grooves. Measurements of the height of conductor layers in the block were made after each layer was wound and ultrasonically seated. Tests conducted using this method, the results of which are shown in Figure 13, indicate that each conductor layer was successfully seated $0.15 \mathrm{~mm}$ into the substrate. Electrical hypot testing at $2 \mathrm{KV}$ potential was performed afterwards, between adjacent wires and between all wires and the grounded groove, to verify that the insulation was not damaged by the ultrasonic welding process.

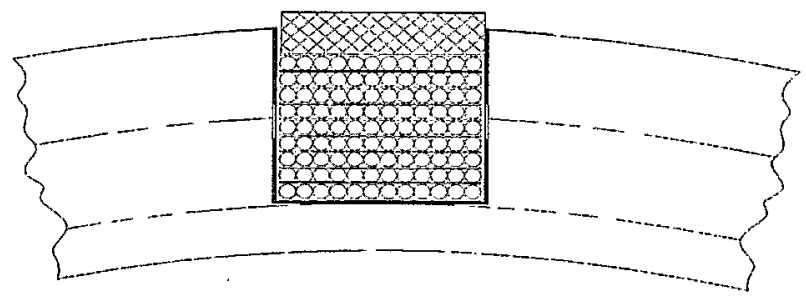

Figure 10. Coil Block cross-section

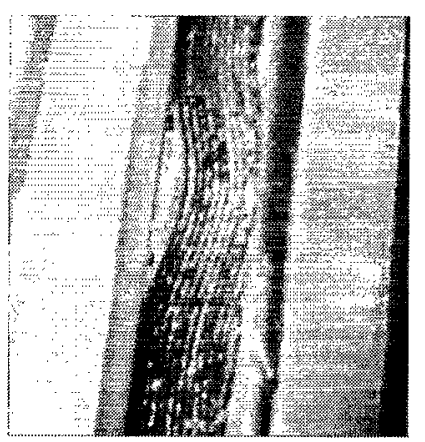

Figure 11. Cable positioning error

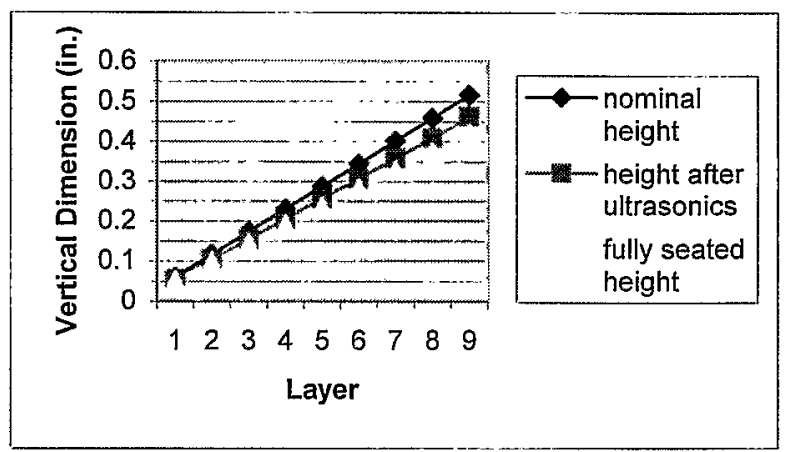

Figure 12. Comparison of Cable Seating Depths

\section{SUMMARY}

Improved methods and tools are being utilized for the design, engineering, and assembly of the AGS Snake magnet coils. These methods and tools will ensure that the coils meet all magnetic, electrical, and mechanical requirements.

\section{REFERENCES}

[1] R.Gupta, et al, "Magnetic design of a Superconducting AGS Snake," this conference. 